\title{
Monitoramento Estatístico Multivariado e de Desempenho de Malhas de Controle de uma Caldeira
}

\author{
Carmela M. P. Braga*, Anísio R. Braga*, Bruno M. Sousa* \\ *Universidade Federal de Minas Gerais (e-mails: carmela@ufmg.br, anisio@ufmg.br,b-sousa@ufmg.br)
}

\begin{abstract}
Thermoelectric power plants have critical units, e.g. boiler that is a complex multivariable system in itself. Such complex units present non-stationary behavior and multiple points of operation, which implies constant changes in variables' setpoints. This work presents a multivariate statistical monitoring methodology, combined with Principal Component Analysis (PCA), to detect changes in operational conditions, adapted to changing process conditions, since strict PCA technique requires stationarity that is not ensured. In addition, performance indices of regulated control loops are used to assess changes in dynamical behavior, which also indicates degradation of a boiler-unit performance. The proposed methodology was implemented in a PIMS environment, integrating a monitoring system, installed in a thermoelectric power plant used as a case study. Experimental results based on actual operational data from the case study power plant are given to illustrate results of the proposed methodology.
\end{abstract}

Resumo: Usinas termelétricas (UTEs) possuem unidades críticas, como caldeira, que é uma unidade multivariável complexa em si. Tais unidades apresentam comportamento não estacionário e múltiplos pontos de operação, o que implica mudanças constantes de referências de variáveis de processo. Apresenta-se uma metodologia de monitoramento estatístico multivariado, combinado com análise de componentes principais, para detecção de mudanças nas condições operacionais, adaptada às condições do processo, uma vez que o uso destas técnicas requer estacionariedade e este não a possui. Além disso, são utilizados índices de desempenho de malhas de controle para auxiliar na indicação de mudanças no comportamento das mesmas e no rastreamento das variáveis mais influentes na degradação de desempenho da caldeira. A metodologia proposta foi implementada numa plataforma PIMS, integrando um sistema de monitoramento, instalado numa UTE usada como estudo de caso. Resultados experimentais com dados de operação da usina estudada validam a proposição e ilustram a metodologia.

Keywords: Hotelling's $\mathrm{T}^{2}$ control chart, SPE, PCA, Combined index, Multivariate Statistical Process Control, Control loop performance monitoring, Boiler.

Palavras-chaves: Carta de controle de Hotelling $\mathrm{T}^{2}$, SPE, Índice combinado, PCA, Controle Estatístico de Processos Multivariados, Monitoramento de desempenho de malhas, Caldeira.

\section{INTRODUÇÃO}

Usinas termelétricas, apesar de serem mais custosas no que se refere a operação e manutenção, possuem um importante papel de estabilização da matriz energética brasileira, a qual é baseada principalmente na hidroeletricidade e, portanto, fortemente dependente de mudanças nas características climáticas (ANEEL, 2002; Sousa et al., 2015). Contudo, o aumento na queima de combustíveis fósseis para geração termelétrica como fonte complementar de energia em períodos de seca tende a piorar as mudanças climáticas associadas à emissão de gases do efeito estufa. Deste cenário emerge forte demanda por ferramentas e sistemas automatizados que suportem a operação e manutenção de usinas termelétricas em um alto nível de eficiência, com máximo índice de disponibilidade e poluição parcimoniosa (Sousa et al., 2015).
Unidades críticas de usina termelétrica, e.g. caldeira, são sistemas complexos que integram variáveis impactantes na eficiência global (Åström; Bell, 2000). Usinas que funcionam como fornecedoras de energia complementar e que utilizam diferentes combustíveis geralmente possuem a característica de multiplicidade de pontos operação e de não estacionariedade em torno desses pontos. Tais condições resultam em alterações frequentes nas referências das variáveis chave e, portanto, limitam o uso de técnicas clássicas de Controle Estatístico de Processos (CEP), por causa do requisito de estacionariedade. Além disso, a ocorrência de faltas ou degradações incipientes afeta, em geral, mais de uma variável simultaneamente. Assim, considerando o grande número de variáveis monitoradas que possivelmente apresenta correlações com faltas, há necessidade de métodos capazes de lidar tanto com a correlação entre elas - como os métodos de Controle Estatístico de Processos Multivariados (CEPM) - quanto com seu comportamento inerentemente quase ou não estacionário. 
Duas importantes ferramentas estatísticas de análise e monitoramento multivariados são, respectivamente, a técnica de Análise das Componentes Principais (PCA, do inglês, Principal Component Analysis) e a carta $\mathrm{T}^{2}$ de Hotelling. $\mathrm{O}$ monitoramento com carta de Hotelling baseia-se na tradução das informações de um conjunto de variáveis correlacionadas em um único índice estatístico, o T² (Mason; Young, 2002). Esse índice é calculado de acordo com (1), em que X é o vetor amostral de variáveis com observações unitárias. Ainda em (1), $\overline{\boldsymbol{X}}$ e $\boldsymbol{S}$ são, respectivamente, o vetor de médias das variáveis e a estimativa da matriz de covariância do sistema quando este opera em estado de controle estatístico.

$$
T^{2}=(\boldsymbol{X}-\overline{\boldsymbol{X}})^{\mathrm{T}} \boldsymbol{S}^{-1}(\boldsymbol{X}-\overline{\boldsymbol{X}}) .
$$

De acordo com Montgomery (2009), a carta de Hotelling perde sensibilidade à medida que o número de variáveis $p$ aumenta, já que uma possível alteração na média de um processo é dissolvida no espaço $p$-dimensional e, por isso, incorre na possibilidade de ser não detectada. A combinação PCA-CEPM é justificada na medida em que a técnica de PCA permite a redução do espaço de variáveis sem que haja perda significativa de informação, gerando um conjunto menor de componentes principais que incorpora a maior parte da variabilidade do sistema. A aplicação do índice $\mathrm{T}^{2}$ sobre as componentes principais retidas fornece uma métrica do desempenho global do sistema. Adicionalmente, a análise das componentes principais na ocorrência de pontos fora dos limites de controle da carta provê suporte ao rastreamento de faltas, já que os scores, com maior influência no alarme, indicam quais variáveis contribuíram mais fortemente para que o processo saísse do estado de controle estatístico.

Outros índices estatísticos multivariados são frequentemente utilizados para a detecção de alterações no comportamento dos processos (Pessel; Balmat; Lafont; Bonnal, 2007; Wang; $\mathrm{Ma} ; \mathrm{Xu}, 2011$ ). O SPE (do inglês, squared prediction error), em especial, é capaz de medir o desvio entre o conjunto original dos dados e a predição das variáveis feita a partir do modelo resultante do PCA. Na prática, o monitoramento do processo por meio de um único índice é preferível pelas equipes de operação e manutenção. Yue e Qin (2001) propõem um índice combinado $\varphi$, que sumariza informações tanto do $\mathrm{T}^{2}$ quanto do SPE. Logo, um valor de $\varphi$ que ultrapasse os limites de controle deve ser seguido da inspeção individual tanto do índice $\mathrm{T}^{2}$ quanto do SPE.

Além dos índices estatísticos multivariados, monitorar o desempenho das malhas de controle beneficia equipes envolvidas propiciando informação adicional acerca do estado de controle regulatório do processo. Além de verificarem se a operação da malha está dentro de níveis aceitáveis, métricas de desempenho dinâmico fornecem suporte para detectar e apontar situações que requerem novas sintonias, ou mesmo a substituição de componentes de instrumentação. Dos vários índices de desempenho encontrados tanto na literatura científica quanto nos manuais de softwares comerciais (Campos; Teixeira, 2010; Controlsoft, 2019; Jota; Braga; Pena, 1995; Metso Expertune, 2019), este trabalho aplica três: a integral do erro absoluto
(IAE), a variabilidade (desvio padrão) da variável controlada (VarY) e a variabilidade da variável manipulada (VarU).

Alguns resultados significativos do uso de técnicas de CEPM para monitoramento de variáveis de processo têm sido publicados. Zhu e Braatz (2014) propõem, em conjunto com as técnicas de PCA e com a carta de Hotelling T², a aplicação de um mapa de contribuição bidimensional para a identificação de faltas em processos multivariados. Os autores obtêm resultados positivos com a aplicação dessas ferramentas sobre dados do Tennessee Eastman Process, modelo matemático de referência (benchmark) para estudos simulados de processos de uma indústria química. Qin (2003) discute a utilização da carta de Hotelling, da técnica de PCA e de outros métodos multivariados para a detecção, identificação e diagnóstico de faltas. Lee e outros (2006) utilizam o método de independent component analysis no monitoramento de um processo de tratamento de água.

Este trabalho apresenta a implementação combinada das técnicas de PCA-CEPM para detecção de mudanças nas condições operacionais de uma usina termelétrica e auxílio no rastreamento de faltas por meio do PCA, como suporte à gestão de ativos e ao monitoramento de desempenho. São consideradas seções de uma unidade de caldeira consideradas críticas para o desempenho global. A usina em questão possui ambas as características mencionadas: múltiplos pontos de operação e não estacionariedade, que podem ser explicadas pelas variações na demanda energética exigida e na composição e disponibilidade dos combustíveis. Como as técnicas de PCA-CEPM requerem comportamento de quaseestacionariedade, é proposto um mecanismo de adaptação de média para a padronização das amostras que possibilita que os diferentes pontos de operação da usina possam ser adequadamente tratados pelos métodos estatísticos.

$\mathrm{O}$ sistema de monitoramento é um apoio às equipes de operação e manutenção da usina cujo objetivo é (i) alertar sobre alterações inesperadas nas condições de operação da caldeira sem avalanche de alarmes, (ii) auxiliar no rastreamento de faltas e (iii) formatar os dados em termos de índices estatísticos e índices de desempenho de malhas de controle para criação de banco de conhecimento do processo. Esses recursos viabilizam uma operação mais ciente do desempenho da caldeira e suas malhas de controle e de possíveis degradações incipientes. Propiciam, também, ações da engenharia de manutenção proativa, capazes de antever e programar intervenções sobre equipamentos e instrumentos quando há indícios fortes de degradação e/ou falta incipiente.

A seção 2 apresenta uma descrição do estudo de caso da usina de maneira geral, destacando os aspectos mais importantes para o desenvolvimento do trabalho e detalhando as unidades mais críticas para o desempenho, sendo este trabalho voltado para o monitoramento da caldeira. A seção 3 apresenta a metodologia empregada no monitoramento das seções críticas, enquanto que a seção 4 mostra os resultados obtidos e as respectivas análises. Por fim, a seção 5 sumariza as conclusões do trabalho. 


\section{USINA TERMELÉTRICA DO ESTUDO DE CASO}

A usina termelétrica considerada neste estudo de caso foi projetada para operar com três tipos de combustíveis: gás de alto-forno, gás natural e alcatrão conforme ilustrado no digrama da Fig.1. Cada um dos combustíveis apresenta poder calorífico diferente e tal diferença tem impacto direto na dinâmica das variáveis controladas e na eficiência da usina. Portanto, é necessário que o operador manipule a quantidade de alcatrão e de gás de alto-forno disponibilizada para queima, complementada pela de gás natural que, embora sempre disponível, tem custo superior aos outros dois combustíveis supracitados. Nesse sentido, ao manipular a quantidade e a proporção de cada um dos combustíveis, o operador está, na verdade, alterando o ponto de operação da usina a fim de otimizar a eficiência da mesma.

$\mathrm{O}$ vapor superaquecido gerado na caldeira é direcionado à turbina, que converte energia térmica do vapor em energia cinética através do movimento de rotação de suas pás (Woodruff; Lammers; Lammers, 2005). A velocidade de rotação é proporcional à diferença de temperatura do vapor na entrada e na saída da turbina. À medida que o vapor perde energia ele é retirado da turbina através de quatro pontos de extração e reutilizado em processos de pré-aquecimento de combustíveis e água. Um gerador acoplado ao eixo de rotação da turbina é responsável pela conversão da energia cinética em energia elétrica, e a potência gerada depende da demanda exigida pela empresa proprietária.

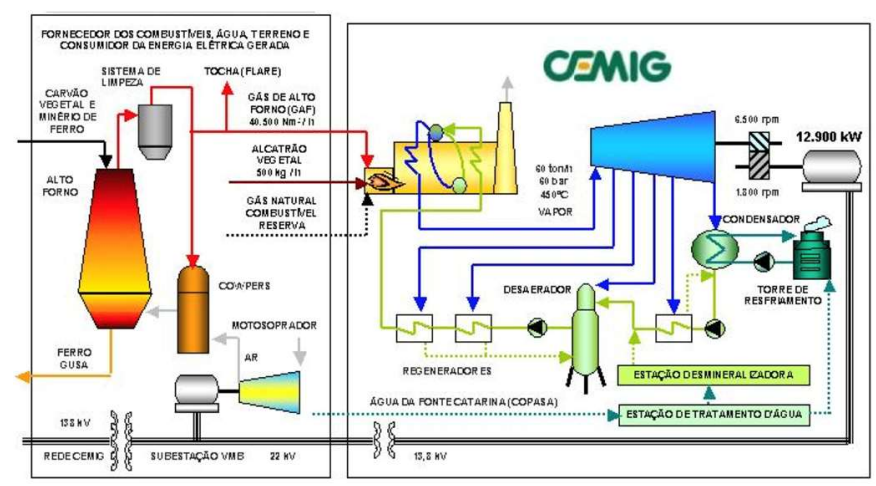

Fig. 1. Diagrama da usina do estudo de caso.

\section{METODOLOGIA}

A metodologia de PCA-CEPM que trata as condições de não estacionariedade ou de quase-estacionariedade consiste em três etapas principais: (i) pré-filtrarem, correção de inconsistências nas amostras e determinação da região de referência ou nominal do processo; (ii) cálculo das componentes principais, bem como o projeto das cartas de Hotelling $\mathrm{T}^{2}$ e dos índices SPE e combinado; (iii) validação das cartas através do monitoramento de um novo conjunto de dados. Tais procedimentos são detalhados nas subseções seguintes.

\subsection{Tratamento Inicial dos Dados e Seleção das Regiões de Projeto}

Inicialmente, deve-se determinar a lista das variáveis de maior interesse para monitoramento e controle estatístico de cada unidade do processo, neste caso em particular, a caldeira. Isso é feito por meio da análise de fluxogramas de engenharia $(P \& I D)$ do processo e da consideração dos conhecimentos dos operadores, técnicos e engenheiros envolvidos. Uma vez coletados os dados, deve-se verificar a integridade dos arquivos e a consistência das amostras. Isso significa, principalmente, interpolar dados faltantes e remover mensagens esporádicas de falhas. A carta de Hotelling $\mathrm{T}^{2}$ requer que os dados estejam sincronizados e que haja uniformidade no intervalo de amostragem. Nesse trabalho, os dados foram retirados de um sistema PIMS com uma taxa de amostragem de $1 \mathrm{~Hz}$.

Após a pré-filtrarem e a verificação da consistência das amostras, o próximo passo é realizar a dizimação dos dados. A dizimação é importante para minimizar alarmes excessivos da carta de controle na etapa de monitoramento do processo. A determinação da taxa de dizimação é feita a partir do método de tentativa e erro combinado com inspeção visual dos dados, com o intuito de reduzir o número de amostras sem ocasionar perdas significativas de dinâmicas dominantes e de mudanças abruptas dos dados.

De modo semelhante à etapa de escolha da taxa de dizimação, é feita a inspeção visual das variáveis principais da unidade caldeira, a fim de determinar os segmentos em que o processo opera em estado de controle estatístico, aqui denominados de regiões de projeto. Operadores e engenheiros do processo são consultados para auxiliar na classificação dos segmentos como condições ou patamares de operação de referência ou nominais.

Posteriormente, todas as variáveis são normalizadas usando a média e o desvio padrão de cada região de controle. A normalização das variáveis é de suma importância para o PCA, pois a diferença de escala das variáveis gera resultados inconsistentes relativos à contribuição para a variabilidade de sistemas multivariados (Montgomery, 2009).

\subsection{Projeto da Carta de Hotelling $T^{2}$, Definição dos Limites dos Índices SPE e $\varphi$ e Análise das Componentes Principais}

$\mathrm{O}$ vetor de scores $\boldsymbol{z}$ das componentes principais de um conjunto original de $p$-variáveis, $\boldsymbol{X}$, é calculado como:

$$
\boldsymbol{z}=\boldsymbol{U}^{\mathrm{T}},
$$

em que as colunas da matriz $\boldsymbol{U}$ são os autovetores da matriz de autocorrelação do sistema. Os autovetores que compõem a matriz $\boldsymbol{U}$ representam as principais direções de variabilidade dos dados, ou das componentes retidas.

Nos estudos de caso apresentados neste trabalho, é calculada uma matriz de correlação $\boldsymbol{U}$ para cada uma das regiões de projeto da caldeira, uma vez estas apresentam estatísticas distintas. Na fase de projeto da carta de Hotelling, o índice $\mathrm{T}^{2}$ 
é calculado usando as componentes principais que explicam a maior parte da variabilidade do sistema (95\%):

$$
T^{2}=\left(\mathbf{z}_{r}-\overline{\mathbf{z}}\right)^{\mathrm{T}} \boldsymbol{S}_{z}^{-1}\left(\mathbf{z}_{r}-\overline{\mathbf{z}}\right),
$$

em que $\boldsymbol{z}_{r}$ e $\overline{\boldsymbol{z}}$ são, respectivamente, o vetor de scores e o vetor com as médias dos scores retidos. $\boldsymbol{S}_{z}$ é a matriz de covariância dos scores. O SPE e o índice combinado são calculados de acordo com (4) e (5), respectivamente:

$$
\begin{gathered}
S P E=\left(\mathbf{z}_{l}\right)\left(\mathbf{z}_{l}\right)^{T}, \\
\varphi=\frac{T^{2}}{T_{\alpha}^{2}}+\frac{S P E}{Q_{\alpha}},
\end{gathered}
$$

em que $l$ é o número de componentes descartadas e $\boldsymbol{z}_{l}$ é o vetor com as componentes descartadas. Esse método de cálculo é uma alternativa àquele referenciado por (Jackson, 2003). $T_{\alpha}^{2}$ e $Q_{\alpha}$ são, respectivamente, os limites da carta de Hotelling $\mathrm{T}^{2}$ e do índice SPE encontrados na etapa de projeto.

Os limites de controle, tanto para a fase de projeto quanto para a fase de monitoramento, dependem do número de pontos das regiões de projeto e do número de componentes principais retidas (Mason; Young, 2002; Montgomery, 2009).

\subsection{Monitoramento}

Na etapa de monitoramento, os parâmetros gerados na fase de projeto são usados para realizar o monitoramento online do processo por meio da carta de Hotelling $\mathrm{T}^{2}$, do SPE e do índice combinado. Além disso, são monitorados os seguintes índices de desempenho de malhas: IAE, variabilidade da variável controlada (VarY) e variância da variável manipulada (VarU). A Tabela I destaca todas as malhas monitoradas. Os cálculos do IAE, de VarY e VarU para cada instante $k$ são dados por (6) e (7) (Sousa, 2015, Jota; Braga; Pena, 1995):

$$
I A E[k]=\operatorname{IAE}[k-1]+\alpha(|e[k]|-I A E[k-1]) .
$$

As estimativas da variância da variável manipulada e da variável controlada, respectivamente, no instante $k$, foram calculadas usando o algoritmo (7):

$$
\left\{\begin{array}{c}
v_{m}[k]=v_{m}[k-1]+\beta\left(v[k]-v_{m}[k-1]\right) \\
V_{m}[k]=V_{m}[k-1]+\beta\left(v^{2}[k]-V_{m}[k-1]\right) \\
\operatorname{Var}_{v}[k]=\sqrt{\frac{2-\alpha}{2(1-\alpha)}\left(V_{m}[k]-v_{m}^{2}[k]\right)}
\end{array}\right.
$$

Fazendo-se $v[k]=U[k]$ para obter variabilidade da variável manipulada, e $v[k]=Y[k]$ para a variabilidade da variável controlada.

Em (6), $\alpha$ é um fator de esquecimento: quanto maior seu valor, mais se prioriza a entrada atual no cálculo do IAE e menor é o peso dado ao valor passado da saída. A mesma interpretação é usada para $\beta$ em (7).

A Fig. 2 apresenta um diagrama de blocos do algoritmo de PCA-CEPM com foco na etapa de monitoramento. As amostras multivariadas devem ser coletadas a uma taxa correspondente à taxa de dizimação empregada na fase de projeto. Uma vez coletadas as amostras, elas são normalizadas para evitar que diferenças nas escalas gerem valores incorretos no momento do cálculo do PCA. Entretanto, como a usina apresenta mudanças frequentes de setpoint e múltiplos patamares de operação, realizar a normalização usando a média da região de projeto é uma abordagem limitada e que pode produzir resultados inconsistentes. Uma solução para esse problema é fazer a padronização das amostras usando um estimador de média adaptativo ao invés de utilizar a média da região de operação considerada (Sousa et al., 2015).

Tabela I. Malhas de controle monitoradas.

\begin{tabular}{|l|}
\hline \multicolumn{1}{|c|}{ Malha de controle } \\
\hline Vazão GAF na entrada do queimador \\
\hline Vazão de água de alimentação \\
\hline Pressão do vapor no superaquecedor \\
\hline Temp. do vapor no superaquecedor \\
\hline Vazão de ar de combustão \\
\hline Nível de água do tubulão \\
\hline Pressão do ar de combustão \\
\hline Pressão do GAF \\
\hline Pressão na câmara de queima \\
\hline
\end{tabular}

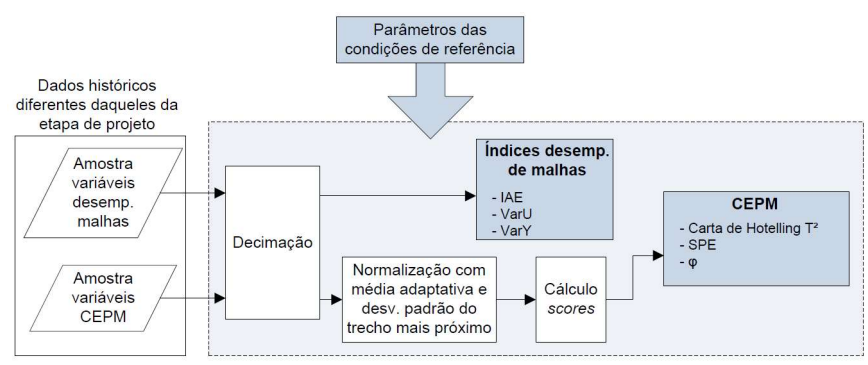

Fig. 2. Diagrama de blocos do algoritmo de monitoramento dos índices multivariados e do desempenho das malhas de controle.

O estimador adaptativo de média provê um mecanismo rápido de adaptação quando ocorrem mudanças de referência ou comandos do operador (Sousa et al., 2015). Para isso, a amostra de cada uma das variáveis é entrada de dois filtros de média aritmética recursivos. Um filtro de média rápida, que acompanha melhor as variações dos dados originais, e o outro de média lenta, que apresenta dinâmica mais lenta em relação ao sinal original. Sobre o resíduo das saídas desses filtros é aplicado um teste CUSUM (Cumulative Sum), que detecta mudanças abruptas ou comandos do operador e envia um comando para que a saída do filtro lento seja chaveada para o valor da saída do filtro rápido. A saída do filtro lento é usada para selecionar os parâmetros do ponto de operação parâmetros do PCA e limites de controle - e normalizar os dados. Dessa forma, o algoritmo PCA-CEPM foca na variabilidade do processo durante o período de monitoramento como se este fosse quase estacionário. As componentes principais retidas são usadas para monitorar aproximadamente $95 \%$ da variabilidade dos dados com um intervalo de confiança de $99 \%$. 
A escolha das variáveis da caldeira a serem monitoradas utilizando técnicas de CEPM foi baseada, principalmente, no estudo da operação do processo, na análise de diagramas de engenharia e na experiência de operadores e engenheiros envolvidos no projeto. A Tabela III apresenta a relação das variáveis selecionadas. Em paralelo às variáveis da caldeira, os índices de desempenho das malhas de controle também são monitorados.

\section{Tabela III. Relação de variáveis da seção da caldeira de} maior relevância para o monitoramento estatístico.

\begin{tabular}{|l|l|}
\hline \multicolumn{1}{|c|}{ TAG } & \multicolumn{1}{c|}{ Descrição } \\
\hline FIQ101 & $\begin{array}{l}\text { Vazão de gás de alto-forno na entrada do } \\
\text { queimador }\end{array}$ \\
\hline FIQ102 & Vazão de gás natural na entrada do queimador \\
\hline FIQ313 & Vazão de água de entrada \\
\hline FIT301 & Vazão de vapor superaquecido \\
\hline PI343 & Pressão de vapor no tubulão \\
\hline PIC322 & Pressão do vapor superaquecido \\
\hline TIC327 & Temperatura do vapor superaquecido \\
\hline TI323 & Temperatura da água de alimentação \\
\hline TI329 & Temperatura dos fumos (gases de exaustão) \\
\hline TI343 & Temperatura do gás de alto-forno \\
\hline FIQ311 & Vazão de ar para combustão \\
\hline AIC301 & Análise de oxigênio dos gases de exaustão \\
\hline
\end{tabular}

\section{RESULTADOS}

O conjunto de dados da caldeira utilizado para o projeto da carta de Hotelling corresponde a um período de dados de 4 dias em maio e 7 dias em julho de 2015. Esse recorte foi realizado com a finalidade de privilegiar períodos em que a usina operava em patamares de potência diferentes e bem definidos, de modo que a tarefa de seleção dos trechos de referência para cada patamar fosse facilitada. Como os pontos de operação variam consideravelmente, foram escolhidos quatro trechos que representam o comportamento típico da usina para o projeto em cada patamar típico de operação, considerando-se que são também representativos do estado de controle estatístico dos mesmos. A Fig. 3 apresenta o período de dados de potência ativa, com destaque para os trechos de projeto escolhidos. O número de componentes principais retidas em cada trecho de projeto busca explicar cerca de $95 \%$ da variabilidade total desses dados.
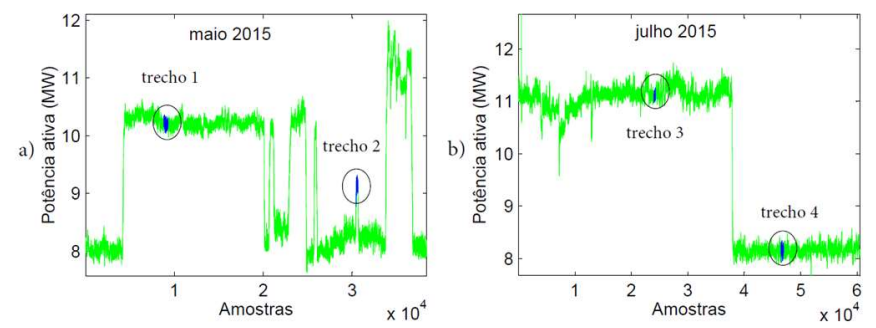

Fig. 3. Trechos escolhidos para o projeto da carta de Hotelling $\mathrm{T}^{2}$ e para o cálculo dos limites dos índices SPE e $\varphi$. Os dados do mês de maio estão indicados em a) e os do mês de julho em b).
Os limites de controle foram projetados para um intervalo de confiança de $99 \%$, para cada uma das quatro regiões de projeto, correspondentes aos quatro patamares típicos de operação.

Para fins de comparação, a Fig. 4 exibe um trecho de monitoramento do processo em que não há desvios da condição de referência. Nela, a potência gerada pela usina é comparada com o índice combinado.

Os índices $\mathrm{T}^{2}$ e SPE do mesmo trecho são exibidos na Fig. 5. Nota-se que os pontos fora dos limites de controle são pouco frequentes e ocorrem em compatibilidade com o intervalo de 99\%. Já a Fig. 6 mostra os índices multivariados em um segmento de dados em que o processo sai do estado de controle estatístico. Uma análise das contribuições de cada score no índice $\mathrm{T}^{2}$ indica alterações principalmente nas variáveis PIC322, FIQ313 e TI329. Nesse mesmo trecho de dados, oscilações nos índices de desempenho das malhas relacionadas a essas variáveis são observadas, como mostram as Fig. 7 e 8 . A Fig. 9 mostra a variação no SP e na saída da malha de PIC322. Tanto o IAE quanto a variabilidade das variáveis controlada (VarY) e manipulada (VarU) são afetados.

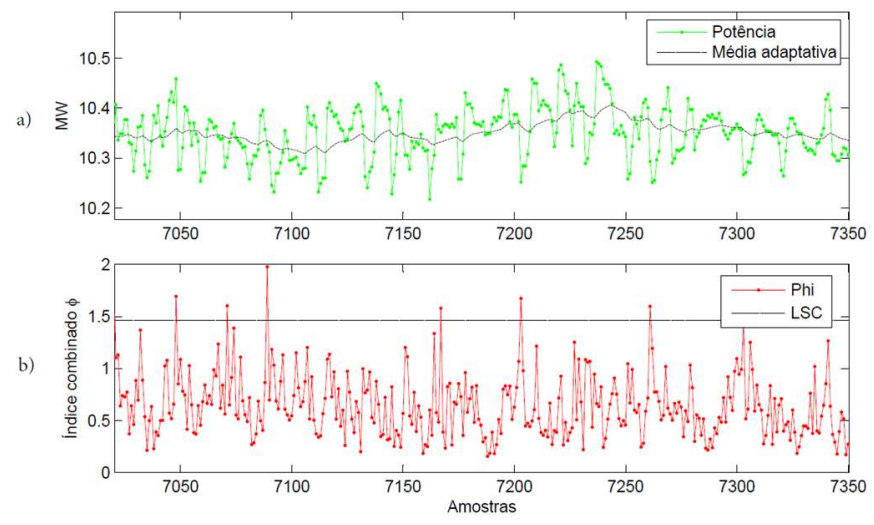

Fig. 4. Potência ativa da usina e índice combinado em um trecho onde não há desvios da condição de referência. Em a) também está indicada a média adaptativa utilizada para o cálculo dos índices.
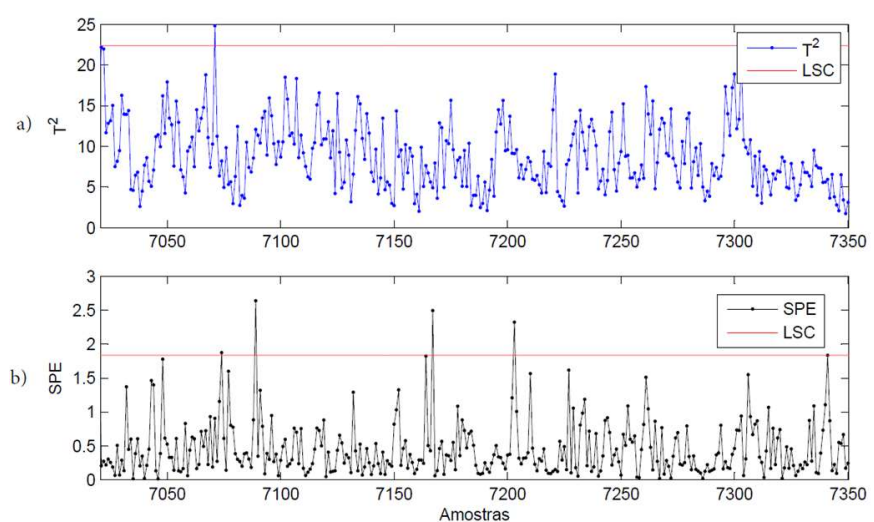

Fig. 5. Índices $\mathrm{T}^{2}$ e SPE do mesmo trecho dos dados exibidos na Fig. 4. Não há a presença de alterações significativas no processo. 

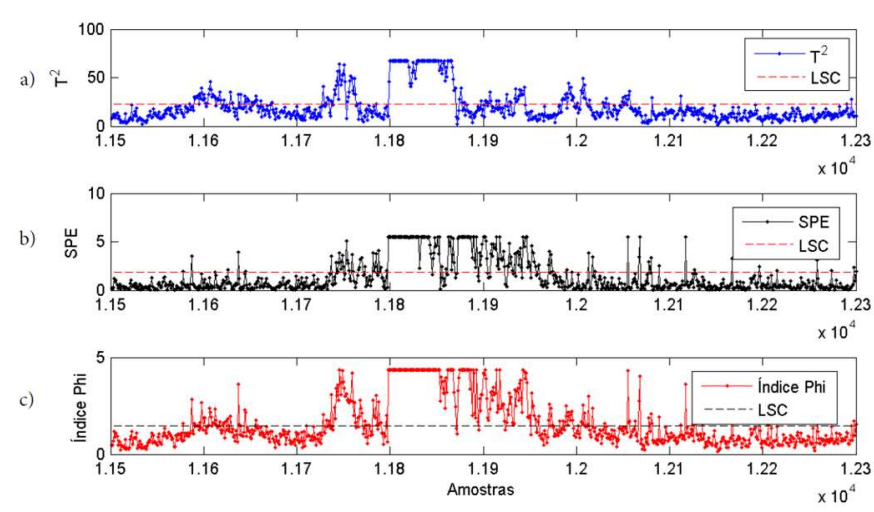

Fig. 6. Índices $\mathrm{T}^{2}$ (a), SPE (b) e combinado (c) em torno das amostras de número 11800 a 11900 indicando a presença de uma condição anormal de operação.

Algumas variáveis com contribuições elevadas no SPE e que fazem parte das malhas monitoradas também possuem alterações percebidas pelos índices de desempenho. É o caso da vazão de GAF (FIQ101), do nível de água no tubulão (LIC302) e da vazão de ar de combustão (FIQ311). Os índices da malha de nível de água no tubulão, por exemplo, são mostrados na Fig. 10. A Fig. 11 exibe alterações em algumas das variáveis apontadas, as quais possuem grande influência na geração de alarmes tanto por meio do índice $\mathrm{T}^{2}$ quanto pelo SPE.
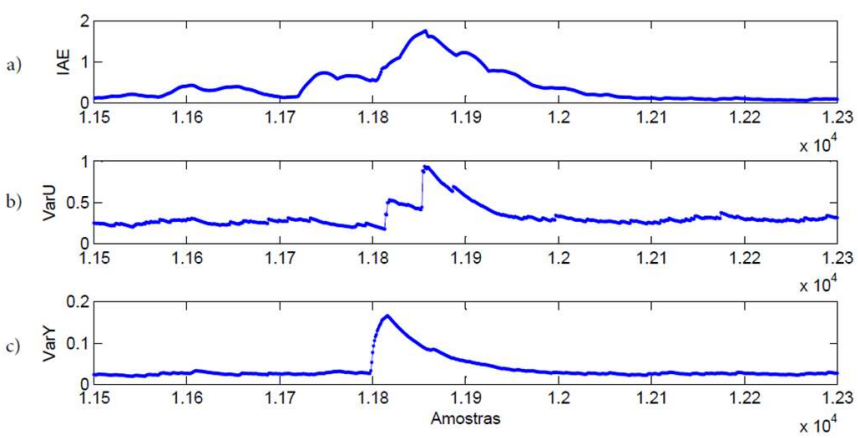

Fig. 7. Oscilação na pressão de vapor superaquecido (PIC322) refletida nos índices da malha de controle: IAE (a), VarU (b) e VarY (c).

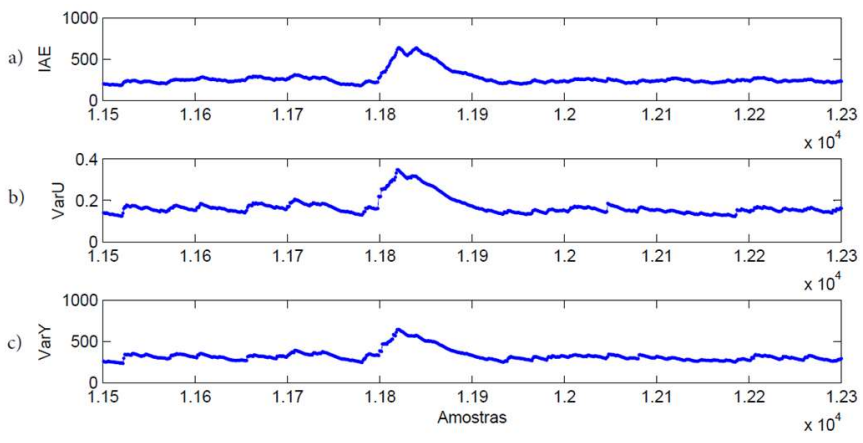

Fig. 8. Oscilação na vazão de água de entrada (FIQ313) refletida nos índices da malha de controle: IAE (a), VarU (b) e $\operatorname{VarY}(\mathrm{c})$.

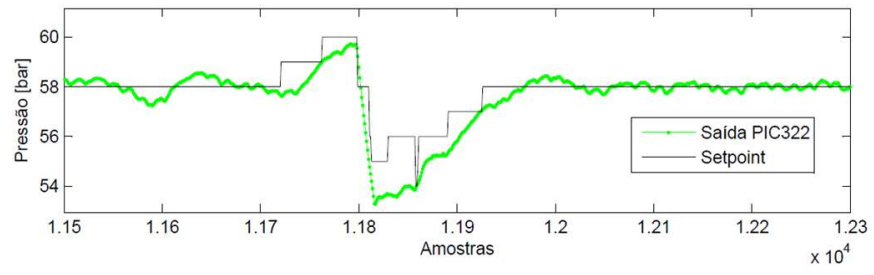

Fig. 9. Variável controlada e setpoint (SP) da malha de vazão de vapor superaquecido (PIC322).

Uma provável análise das alterações observadas começa por considerar o leve aumento, seguido de queda repentina, na pressão do vapor superaquecido. Tal flutuação impacta diretamente na pressão de vapor do tubulão, já que a linha de saída de vapor do tubulão está diretamente conectada à de vapor superaquecido. A queda brusca na pressão dentro do tubulão gera um aumento imediato no nível aparente de água em razão do crescimento do volume das bolhas de vapor. Porém, logo em seguida, como a pressão mais baixa favorece a passagem da água para o estado gasoso, o nível de água apresenta declínio. Essa redução no nível gera aumento na vazão de água de alimentação e de combustíveis, buscando reequilibrar o nível de água e as características de pressão e temperatura do vapor principal. É necessário destacar que o diagnóstico correto das causas do desequilíbrio na pressão de vapor relacionadas a toda essa sequência de alterações na caldeira deve ser feito em conjunto com os operadores, técnicos e engenheiros da usina (Sousa, 2015).

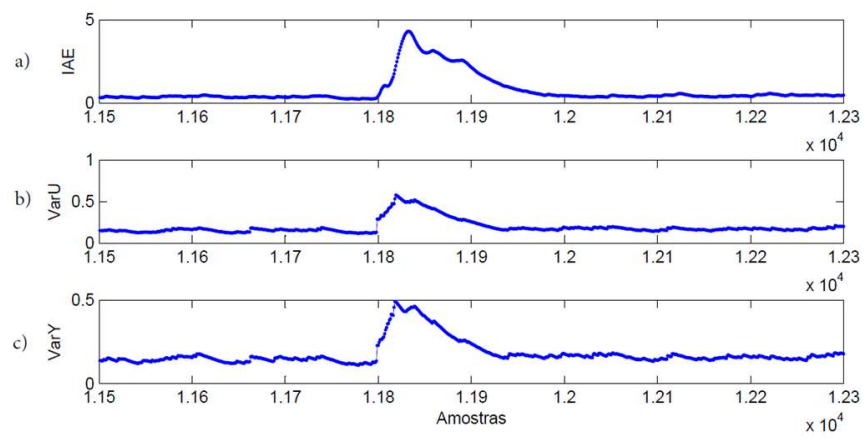

Fig. 10. Oscilação no nível de água do tubulão (LIC302) refletida nos índices da malha de controle: IAE (a), VarU (b) e $\operatorname{VarY}$ (c).

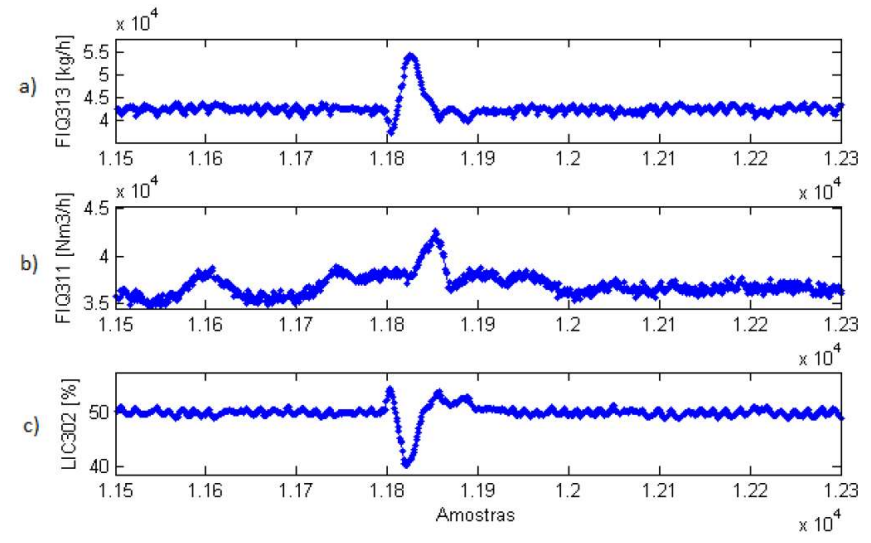

Fig. 11. Alterações observadas na vazão de água de entrada (FIQ313), na vazão de ar de combustão (FIQ311) e no nível de água do tubulão (LIC302). 


\section{CONCLUSÕES}

Foi apresentado o uso de técnicas de controle e monitoramento estatístico multivariado, com um esquema proposto de adaptação de média para adequação às condições de operação não-estacionárias e com múltiplas referências. A aplicação da metodologia proposta, considerando as fases de projeto e monitoramento para a caldeira, que opera com múltiplas referências, demonstra a validade da metodologia proposta. Demonstrou-se que o uso combinado dessas técnicas com o monitoramento dos índices de desempenho de malhas é capaz de detectar mudanças nos casos em que há alteração não usual no processo, provendo às equipes de operação maior agilidade no relato dessas condições.

O uso simultâneo de Índices de avaliação de desempenho para as malhas de controle contribui para confirmar a influência de mudanças no desempenho das malhas, nas situações em que os índices estatísticos indicam perda do estado de controle estatístico, ou ainda, degradação das condições operacionais. A engenharia de manutenção pode se beneficiar, também, com a análise sistemática dos resultados do monitoramento, pois estes proporcionam conhecimento sobre o desempenho do processo e do sistema de automação, sobre a correlação entre as variáveis e os problemas ocorridos, além de prover informação prévia sobre degradações em curso e em qual local. Esta informação prévia sobre possíveis degradações pode tornar a manutenção mais proativa, planejando uma intervenção sobre um sistema, um instrumento ou equipamento enquanto a degradação ainda não implica em falha, evitando que esta venha a ocorrer. No caso de alguma malha de controle estar envolvida, uma auditoria dessa malha mostra-se desejável, uma vez que a degradação do seu desempenho pode estar associada a problemas com o medidor, o atuador ou a sintonia do controlador.

Assim sendo, pode-se dizer que a técnica combinada proposta de PCA-CEPM com média adaptativa, em conjunto com o monitoramento do desempenho das malhas, pode ser encapsulada como uma ferramenta auxiliar para monitoramento em tempo hábil de processos multivariados não-estacionários e com múltiplos pontos de operação, apoiando as equipes de operação e manutenção a manterem a planta operando em suas melhores condições.

\section{REFERÊNCIAS}

ANEEL (2002). Atlas de Energia Elétrica do Brasil. [Online]. Disponível: http://www.aneel.gov.br/arquivos/pdf/livro_atlas.pdf.

Aström, K. J. and Bell, R. D. (2000). Drum-boiler dynamics. Automatica, volume 36 (3), 363-378.

Campos, M. C. M. M and Teixeira, H. C. G. (2010). Controles típicos de equipamentos e processos industriais. Edgard Blucher (2ed).

Controlsoft (2019). INTUNE+ Control Loop Performance Monitoring. [Online]. Disponível em:

$<$ https://www.osisoft.com/marketplace/solutiondetail/?id=a2K1I0000001XgUUAU>. Acesso em: 12 abr. 2019.
Jackson, J. E. (2003). A User's Guide to Principal Components. Wiley Interscience.

Jota, F.G., Braga, A.R., Pena, R.T. (1995). Performance assessment of advanced process control algorithms using an interacting tank system. In $I A S^{\prime} 95$ Conference Record of the 1995 IEEE Industry Applications Conference Thirtieth IAS Annual Meeting, 1565-1571.

Lee, J.-m, Qin, S. J. and Lee, I.-B (2006). Fault Detection and Diagnosis Based on Modified Independent Component Analysis. AIChE Journal, volume 52(10), 3501-3514.

Mason, R. L. and Young, J. C. (2002). Multivariate Statistical Process Control with Industrial Applications. ASA-SIAM.

Metso Expertune (2019). MPC Performance Monitoring. [Online]. Disponível em:

$<$ https://www.metso.com/contentassets/3f1c4763b0db426ea8 fba 833 febe6eb7/mpcperformance-_monitoring.pdf $>$. Acesso em: 12 abr. 2019.

Montgomery, D. C. (2009). Introduction to Statistical Quality Control. John Wiley \& Sons.

Pessel, N., Balmat, J-F., Lafont, F., Bonnal, J. (2007). An Improved PCA Fault Detection for the Diagnosis. In Proceedings of the 9th WSEAS International Conference on Automatic Control, Modeling \& Simulation, 312-318.

Qin (2003), S. J. Statistical Process Monitoring: Basics and Beyond. Journal of Chemometrics, volume 17(8-9), 480502.

Sousa, B. M., Lebedev, A., Ferreira, J., Aguiar, W. E., Braga, C. M. P., Braga, A. R., Lemos, A. P. (2015). Multivariate Statistical Process Control of Boiler and TurbineGenerator units of a Thermoelectrical Power Plant With Multiple Operating Set Points. In Proc. 2015 IEEE International Conference on Industrial Technology, 1907-1913.

Sousa, B. M. (2015). Desenvolvimento de um Sistema para Validação Ampla de uma Caldeira com Múltiplos Pontos de Operação. Dissertação de Mestrado, Dept. Eng. Elétrica, Univ. Fed. Minas Gerais, Belo Horizonte.

Wang, Q., Ma, J., Xu, X., (2011). PCA-AR based Fault Prognosis for Turbine Machine. In Proceedings of the 2011 IEEE International Conference on Mechatronics and Automation, 1605-1610.

Woodruff, E. B., Lammers, H. B., Lammers, T. F. (2004). Steam Plant Operation. McGraw-Hill.

Yue, H. H. and Qin, S. J. (2001). Reconstruction based fault identification using a combined index. Industrial and Engineering Chemistry Research, volume 404403 4414.

Zhu, X. and Braatz, R. (2003). Two-Dimensional Contribution Map for Fault Identification. IEEE Control Systems Magazine, volume 34(5), 72-77. 